\title{
Lectura comprensiva y producción textual: literatura tradicional con apoyo de las TIC
}

Claudia Inés Romero Vaca* Elmi Cruz Pinzón ${ }^{* * *}$

Artículo de investigación

Fecha de Recepción: 23 julio 2018.

Fecha de Aprobación: 4 noviembre 2018.

\section{Resumen}

La propuesta sobre lectura comprensiva y producción textual busca comprobar si al motivar a los estudiantes de los grados tercero a quinto de básica primaria de una sede de la I.E. Técnica de Miraflores, mediante talleres donde se haga recopilación de literatura tradicional, popular del entorno y proporcionar herramientas tecnológicas que permitan recrear las antologías hechas, se logra el desarrollo de habilidades comunicativas para mejorar la producción textual y lectura comprensiva. La investigación presenta un enfoque cualitativo, porque se ha identificado un problema común en la población; la muestra objeto de estudio corresponde a dos estudiantes de grado tercero, siete de cuarto y dos de quinto, orientados por una sola docente, quien, además, debe atender los grados transición a quinto; es una escuela unitaria. Para la selección de la muestra se tuvo en cuenta que los grados tercero y quinto deben presentar prueba saber en este año lectivo; mientras que los estudiantes de grado cuarto presentaron prueba
* Institución Educativa

Técnica Miraflores - Boyacá - Colombia

romerovclaudis@gmail. com

** Institución Educativa

Técnica Miraflores

elmicruzpinzon@yahoo.es

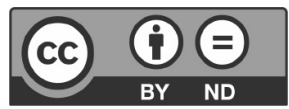


el año anterior con resultados bajos, en el área de lenguaje. La recolección y análisis de información se basa en encuesta realizada a docentes, prueba diagnóstica a estudiantes, y resultados de avances, fortalezas y o dificultades en la aplicación de talleres. Es un proceso flexible que buscar fortalecer procesos básicos de lectura y escritura, en la que se involucran a los integrantes de la comunidad educativa para luego hacer la evaluación de la experiencia

Palabras Clave: lectura comprensiva, producción textual, literatura tradicional. 


\section{Introducción}

La lectura comprensiva y producción textual deben ser procesos creativos e interactivos en construcción permanente, pues son habilidades comunicativas y competencias básicas requeridas para desempeñar con eficiencia cualquier labor. Enseñar a leer y escribir puede parecer muy sencillo, pero esta hipótesis solo tendría validez si este proceso fuera una simple decodificación de símbolos. La mayoría de personas creen que el niño aprende a leer y escribir en grado primero, y es muy común ver cómo los maestros que tienen a cargo esos grados buscan un método mágico que permita lograrlo. Sin embargo, muchos de ellos aún continúan usando el mismo método con el que aprendieron a leer y escribir. En el aula se refleja el poco amor por la lectura; la escasa comprensión y la apatía para construir textos es una constante.

La investigación esta soportada en antecedentes aquí descritos que responden a investigaciones realizadas durante el período comprendido entre los años 2010 y 2015, cuyas categorías tienen pertinencia con las de la presente investigación.

Madero (2013), en su tesis acerca del proceso de comprensión lectora en alumnos de tercero de secundaria, utilizó una investigación mixta secuencial, la investigación se centró en describir el proceso lector que siguen los estudiantes para abordar un texto, con el propósito de comprenderlo. La pregunta fue: ¿Qué proceso siguen los alumnos de tercero de secundaria cuando leen un texto con el propósito de comprenderlo? Esta investigación se trabajó en ocho escuelas, cuatro particulares y cuatro públicas; La investigación permitió construir un modelo que mostraba los pasos que seguían los alumnos al leer un texto con la intención de comprenderlo, la presencia de pensamiento metacognitivo durante el proceso lector y, el uso de estrategias lectoras. La investigación concluyo que los estudiantes en ese nivel tienen claro cuál es el sentido de la lectura, tienen la capacidad de comprender lo que leen y aplican estrategias para dar la intensión a lo que lee.

Duarte (2012), en su tesis doctoral titulada la enseñanza de la lectura como proceso interrelacionado al desarrollo del comportamiento lector, pretendió conocer las prácticas de la lectura desarrolladas por los profesores en la construcción de un pensamiento lector con los alumnos del primer ciclo de aprendizaje de las escuelas públicas principales del municipio de San Luis. Estainvestigación utilizola metodología cualitativa para indagar las prácticas de la lectura y su repercusión en el campo lector. Concluye que la enseñanza de la lectura no debe estar separada de las demás funciones pedagógicas, sino que debe ser un proceso continuo; propone que los docentes creen protocolos que permitan desarrollar prácticas de lecturas enfocadas al autodesarrollo lector y convertir esta actividad en algo rutinario

En el campo nacional se encuentran Betancourth \& Madroñero (2014), en su tesis de maestría La enseñanza
Duarte, R. (2012) en su tesis doctoral titulada la enseñanza de la lectura como proceso interrelacionado al desarrollo del comportamiento lector, pretendió conocer las prácticas de la lectura desarrolladas por los profesores en la construcción de un pensamiento lector con los alumnos del primer ciclo de aprendizaje de las escuelas públicas principales del municipio de San Luis. 
para la comprensión como didáctica alternativa para mejorar la interpretación y producción oral y escrita en lengua castellana en el grado quinto del centro educativo municipal la Victoria de Pasto, el objetivo propuesto es determinar la efectividad de la Enseñanza para la Comprensión como didáctica alternativa para mejorar la interpretación y producción oral y escrita en el grado quinto de la sede principal del Centro Educativo Municipal La Victoria de Pasto. Esta investigación refleja como la poca interpretación y producción textual se convierte en una preocupación de los docentes y en factor que desmejora la adquisición de aprendizajes significativos. Es una oportunidad para que los docentes busquen alternativas que permitan fortalecer dichos procesos, cambiando métodos tradicionales y así tener una mejor calidad de aprendizaje. El proceso investigativo utilizo como estrategia metodológica la implementación del modelo PCK aplicado a una muestra de 16 estudiantes de grado quinto de la sede Central La Victoria en el municipio San Juan de Pasto desarrollando un estudio correlacional con un diseño cuasi experimental.

Los resultados del estudio fueron analizados en tres bloques, uno que exponía del nivel de interpretación, producción oral y escrita que tenían los estudiantes al comenzar el estudio; un segundo bloque describe el nivel que alcanzan los estudiantes muestra después de aplicar la estrategia didáctica y el último bloque es un comparativo entre el momento inicial y el final para cada dimensión de las variables, haciendo evidente el cambio en el desempeño de los estudiantes. Esta investigación concluyó que la enseñanza para el aprendizaje mejora la compresión lectora y la producción textual.

En el ámbito regional se encuentra González (2011) en su tesis maestral Transformaciones en la producción de texto de los niños de grado $2^{\circ}$ primaria a través de la integración de las TIC; esta investigación buscaba responder la pregunta ¿Cómo cambia la producción de textos escritos por los niños de $2^{\circ}$ de básica primaria de la Institución Educativa Técnica Agropecuaria, sede Fuente Toscana municipio de Viracachá con integración de las TIC? El estudio comparó la producción escrita elaborada por niños del grado segundo en el marco de desarrollo de algunas actividades con y sin el uso de herramientas tecnológicas. El objetivo general de esta investigación era Identificar las transformaciones de la producción escrita de textos en la población descrita, concluye que al hacer textos los estudiantes usan el procesador de texto, hacen separación de textos, son más precisos en el uso adecuado de reglas ortográficas $y$ signos de puntuación. Al finalizar la experiencia se comprobó que los niños dejaron el gusto por los juegos de los computadores y mostraron preferencia por escribir usando los procesadores o en el cuaderno de escritura.

El análisis de los antecedentes muestra que el papel del docente debe ser brindar a los estudiantes herramientas para que 
logren desarrollar habilidades de lectura comprensiva, una de las posibles causas de la escasa comprensión lectora puede ser que aún se está enseñando a leer y escribir con los llamados métodos tradicionales, los cuales se limitan a la memorización de sílabas que carecen de significado real, acompañada de la repetición gráfica de algunas planas; en conclusión un método tradicional se limita a la codificación y decodificación de símbolos.

El modelo educativo propuesto en el PEI de la I.E. Técnica Miraflores es el constructivismo, sin embargo, algunos docentes continúan aplicando prácticas tradicionales en el aula. De otro lado, el $95 \%$ de la población escolar de la I.E evidencia dificultades en la compresión lectora, lo que hace que el desempeño de los estudiantes en pruebas internas y externas sea bajo; por consiguiente, con la ejecución de esta propuesta se busca fortalecer el mejoramiento de la competencia de lectura comprensiva induciendo a los estudiantes hacia la lectura crítica desde el uso de la literatura popular y tradicional del entorno.

Esta propuesta busca desarrollar en los estudiantes habilidades en competencias argumentativa, interpretativa y propositiva usando como estrategia la recopilación de la riqueza popular y tradicional oral del municipio de Miraflores para iniciar procesos de lectura comprensiva y producción textual. Por lo anteriormente expuesto se formula la siguiente pregunta de investigación: ¿Cómo influye la recopilación de la literatura tradicional popular de la vereda La Rusa, en el desarrollo de habilidades para mejorar la producción textual y lectura comprensiva en los estudiantes de los grados terceros a quinto de la Institución Educativa Técnica Miraflores del municipio de Miraflores?

Haciendo un análisis del índice sintético de calidad de la I.E del año 2017 y de los resultados de pruebas internas a finales del año 2016, se puede observar el bajo desempeño de los estudiantes de los grados tercero y quinto y, se ha llegado a la conclusión de que la problemática radica en que los estudiantes no hacen procesos de lectura comprensiva y tienen dificultades para proponer $\mathrm{o}$ producir texto.

En la actualidad el mundo gira alrededor de la comunicación y las tecnologías, estas constituyen el telar de lo humano; la comprensión lectora es la base para la adquisición del conocimiento en cualquier área, de ahí la importancia para que el niño potencialice al máximo esta habilidad en los primeros años de escolaridad, para que así tenga un buen desempeño tanto en la vida escolar como social y qué mejor opción que trabajarlo con la ayuda de las TIC. Para que un estudiante sea autor de la construcción de su conocimiento, debe tener la capacidad de leer y su realidad de forma crítica y plasmarla en la producción textual como afirma Freinet en Legrand (2000) "Un día se admitirá que el niño pueda aprender a escribir leyendo y queriendo, saber leer y escribir porque siente la necesidad de este mecanismo cuando está ligado con las experiencias más profundas”.
En la actualidad el mundo gira alrededor de la comunicación y las tecnologías, estas constituyen el telar de lo humano; la comprensión lectora es la base para la adquisición del conocimiento en cualquier área, de ahí la importancia para que el niño potencialice al máximo esta habilidad en los primeros años de escolaridad, para que así tenga un buen desempeño tanto en la vida escolar como social y qué mejor opción que trabajarlo con la ayuda de las TIC. 
Entre las teorías existentes sobre el tema objeto de estudio que sirven como base para profundizar sobre la problemática y la solución de esta, se destacan aquellas que tienen como finalidad exponer la historia o el origen de la escritura, aspectos importantes de las habilidades comunicativas, habilidades comunicativas, analizadas por estudiosos desde diferentes puntos de vista.

Desde un comienzo el hombre se vio en la necesidad de inmortalizar sus ideas, pues la forma más rápida de hacer saber lo que se piensa es hablando, pero este mensaje puede desaparecer tan pronto como se dice. Para que dure un mensaje se debe registrarlo, tallarlo, esculpirlo, estamparlo o imprimirlo. Los primeros mensajes aparecen pintados en las cuevas en la edad de piedra.

En lo concerniente a la producción textual Cassany, en Comas (1993) establece algunas estrategias útiles para componer un texto, ellas son: De igual manera los logros que se pretende alcanzar son la aplicación y el análisis de una encuesta a docentes de la I.E sobre el desempeño de los estudiantes en lectura comprensiva y producción textual; la elaboración y su posterior aplicación de una prueba diagnóstica sobre comprensión lectora y producción textual a los estudiantes de población y muestra; el diseño de talleres que conlleven al rescate de la tradición literaria oral y popular del municipio de Miraflores y, finalmente la evaluación en el avance de cada una de las actividades.

\section{Teorías del conocimiento}

Entre las teorías existentes sobre el tema objeto de estudio que sirven como base para profundizar sobre la problemática y la solución de esta, se destacan aquellas que tienen como finalidad exponer la historia o el origen de la escritura, aspectos importantes de las
- Precisar las ideas sobre la audiencia, el propósito comunicativo y el contexto del texto.

- Planificar la estructura del texto antes de empezar a redactar, de modo que no se pueda modificar según las nuevas ideas que se puedan incorporar en el escrito.

- Hacer la lectura y relectura de lo que se ha planteado o redactado, para mantener el sentido global del texto y así poder verificar si se ajusta a lo que realmente se quiere decir.

- Hacer las correcciones y revisiones, dar un retoque al texto, ayuda definir el contenido final del texto.

- La recursividad permite formular la estructura del texto para modificar los borradores según las nuevas ideas, e incluso rehacerlo de nuevo para tener un texto coherente.

Producir textos es básicamente precisar algunas ideas, estableciendo un propósito comunicativo dentro de un contexto en el texto, y para lograrlo se debe planificar su estructura, leer y releer lo redactado, corregir y revisar aplicando la recursividad. Si el proceso educativo es un aspecto necesario y cultural, debe ser organizado 
$y$ es responsabilidad de docentes crear un ambiente propicio para el desarrollo máximo de las habilidades comunicativas básicas y qué mejor que hacerlo fortaleciendo procesos primarios con la ayuda de las nuevas herramientas tecnológicas.

Para Vygotsky \& Piaget (2012) la adquisición del conocimiento comienza siendo un objeto de intercambio social, y está determinado por la influencia de lo social y la mejor forma de lograr esto es creando espacios donde se le permita al estudiante el fortalecimiento de sus habilidades comunicativas que sirvan de portal para conocer, manipular y utilizar responsablemente las nuevas tecnologías.

Acerca de la lectura y la escritura, se han caracterizado por ser fundamentales en la existencia y en la historia del hombre, pues se han convertido en las habilidades más importantes para el desarrollo social y tecnológico del mundo. La comprensión lectora es la base de la adquisición del conocimiento y la escritura es el complemento de la comprensión. Es evidente que la lectura y la escritura fueron inventadas por diferentes culturas como única manera de transmitir a las generaciones, conocimientos. El hombre siempre ha buscado la manera de expresar en forma escrita lo más relevante de su cultura, inicialmente se crearon los jeroglíficos, y así sucesivamente hasta conformar las letras de los diferentes idiomas y ahora los cibernautas siguen creando códigos comunicativos que mediante el uso de la informática y las tecnologías se han convertido en el nuevo lenguaje universal; todo esto, con el fin de escribir la evolución del ser humano, del ser social y del ser tecnológico de la actualidad.

Así pues, la lectura, la escritura y el uso racional y responsable de las TICS se convierten en un factor determinante en el desarrollo de un pueblo; de ahí la importancia de que nuestros estudiantes estén en la capacidad de comprender lo que se ha expresado a través de la historia de la humanidad, y además la importancia de que el hombre del futuro cree su propio legado cultural y tecnológico.

\section{Materiales y métodos}

En el desarrollo de la propuesta se hizo uso de la metodología investigación acción, definida por Elliott (1993) como "un estudio de una situación social con el fin de mejorar la calidad de la acción dentro de la misma”. La investigación acción se da cuando el investigador no solo quiere identificar un problema sino que busca una forma para solucionarlo, en este caso el investigador debe hacer parte de todas las fases del problema; es quien recolecta la información, interpretación de la misma, planeación y ejecución de propuesta concreta para la solución del problema (Orrego \& Toro, 2014).

Según el modelo de Whitehead enunciado por Latorre (2003) los ciclos de la investigación deben ser sentir o experimentar un problema, para luego imaginar una solución del problema, posteriormente se debe poner en práctica la solución 
imaginada, finalmente se evalúan los resultados de las acciones emprendidas para modificar la práctica a la luz de los resultados.

El enfoque es cualitativo, se parte de la realidad académica de la Institución Educativa, la cual refleja que más del 90\% de la población tiene dificultades para comprender lo que lee, solo logran redactar textos básicos que en la mayoría de las veces son carentes de significado. Es una investigación acción porque tiene como objetivo general solucionar una problemática que aqueja a un alto porcentaje de estudiantes, además los alumnos fueron objetos activos dentro de las estrategias que se aplicaron para la búsqueda de la solución del problema, por otra parte las actividades se diseñaron para que los estudiantes trabajaran en equipo mediante aprendizaje colaborativo.

La población está conformada por 397 estudiantes desde los grados transición hasta grado 11, distribuidos en 17 sedes todas rurales. En la mayoría de ellas se orienta con metodología escuela nueva. La muestra seleccionada para el desarrollo de la propuesta son dos estudiantes del grado tercero; seis de grado Cuarto y dos de grado Quinto de educación básica primaria; para la recolección de información se usa la encuesta a docentes, con el fin de identificar procesos de lectura y escritura dentro del aula; preferencias de los estudiantes, entre otras.

La propuesta se desarrolló en cuatro fases: La primera buscó conocer, comparar y recrear a partir del Libro de recuerdos titulado "Hilando los recuerdos de mis abuelos", las creencias, historias y anécdotas de los abuelos sobre cómo se celebraba la Semana Santa y otras creencias religiosas hace algunos años y compararlas con las vivencias actuales; la segunda fase pretendió rescatar supersticiones, agüeros, refranes, dichos populares de los abuelos, con esta información hacer análisis literal, inferencial y crítico sobre el significado.

En la tercera fase se le concedió libertad al estudiante para indagar sobre su entorno usando lugares conocidos con nombres coloquiales. Finalmente, se hizo la recopilación de frases o palabras que indican sarcasmos $y$ modismos haciendo un análisis crítico de su significado y la afectación que puede producir en el contexto. Toda la información se digitalizo a través de herramientas informáticas que permiten dar a conocer la tradición oral popular del municipio.

Figura 1 Diseño Metodológico de la Investigación

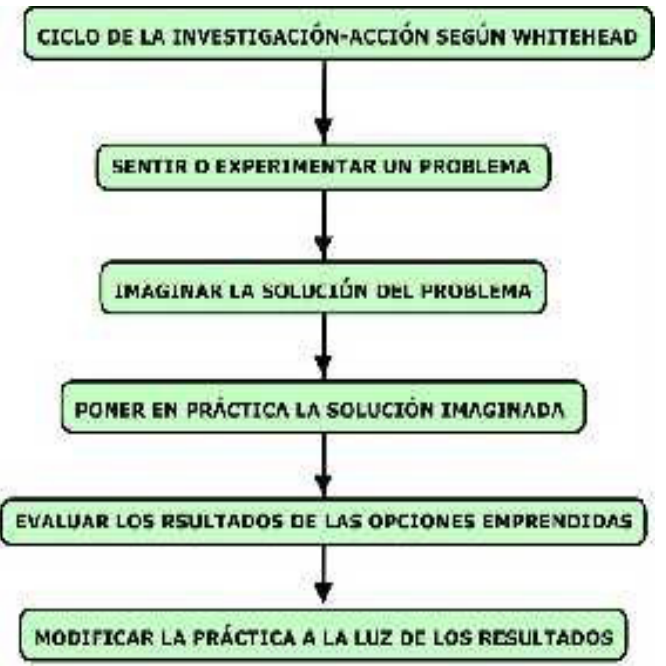

Fuente: los autores 
Se diseñaron y aplicaron talleres para diez estudiantes de los grados tercero, cuarto y quinto de la Sede La Rusa de la Institución Educativa Técnica Miraflores, se hizo uso de la recopilación de la literatura popular de una vereda del municipio de Miraflores y herramientas informáticas como paint, power point, Edilim, entre otras. Estas permitieron recrear las recopilaciones hechas por los estudiantes con el fin de estudiar la influencia que tuvo esta propuesta en el mejoramiento de la lectura comprensiva y la producción textual. La mayoría de estudiantes de estos grados, iniciaron con un nivel bajo en comprensión, pero poco a poco algunos de ellos fueron mejorando el nivel literal, inferencial y solo unos cuantos lograron hacer lectura crítica.

\section{Resultados fase diagnóstica}

La prueba diagnóstica se presentó en tres momentos, el primero contiene trece preguntas sobre el nivel inferencial de una lectura previamente realizada, los estudiantes deben contestar falso o verdadero, el segundo momento está diseñado con diez preguntas abiertas que son contestadas de acuerdo con la comprensión que los niños obtengan luego de leer el cuento y finalmente la producción textual, que consiste en que se realiza una solicitud al estudiante y este deberá darle cumplimiento de la mejor manera posible.

En el análisis del primer momento

En la primera pregunta se pide que el estudiante identifique el motivo de la tristeza de delfincito bebe. Los diez niños que participan en la prueba diagnóstica aciertan al afirmar que la tristeza del delfincito bebe es causada por la pérdida de la mamá, lo que da a entender que los niños comprenden el mensaje de la lectura. En la segunda pregunta, a partir de una afirmación, el estudiante debía inferir la veracidad de la respuesta; seis de los niños logran deducir la respuesta acertada, esto indica que no todos los niños poseen capacidad efectiva de comprender una situación para luego inferir la consecuencia de otra.

En la tercera pregunta se plantea una acción que trae como consecuencia la tranquilidad del delfincito bebe, al igual que la anterior seis de los diez estudiantes logran contestar acertadamente, lo que da a entender una comprensión literal de la lectura, pues la respuesta está escrita dentro del texto que se analiza. A pesar de que en la cuarta pregunta se evidencia una palabra desconocida, para ellos, y una acción que se desprende en una reacción indiferente por parte del delfincito bebe, seis de los niños que participan en la prueba responden consecuentemente. Esto significa que los alumnos comprendieron literalmente el texto leído.

La quinta pregunta muestra acciones de otros animales que deseaban ayudar a solucionar la problemática que presentaba delfincito bebe. La mayoría de los estudiantes recuerdan acertadamente el hilo de la lectura, por tal razón contestan de manera apropiada. En la sexta pregunta se evidencia una condición que debe

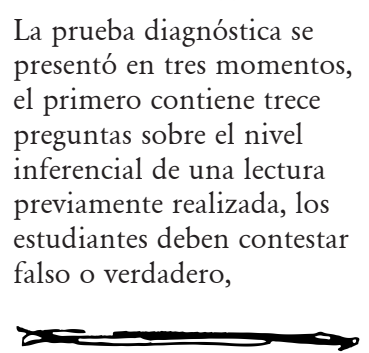

La prueba diagnóstica se presentó en tres momentos, el primero contiene trece previamente realizada, los estudiantes deben contestar 
cumplir el personaje central para que encuentre la solución planteada, al igual que la anterior un alto porcentaje de estudiantes logra recordar del texto lo que sucedía en la mencionada situación.

En la afirmación de la séptima pregunta el estudiante debe verificar, con base en la lectura, la falsedad o veracidad de la misma. La mitad contesta acertadamente, lo que significa que los niños no relacionan el final del cuento con la finalidad de la pregunta, que vendría a ser indagar la comprensión del texto. La octava pregunta propone acciones que posteriormente se desprenden en afirmaciones que el estudiante deberá encontrar a lo largo de la lectura. La mitad de los niños no logran identificar la respuesta, lo que implica que ellos no poseen dominio lector que los conduzca al análisis de las mencionadas afirmaciones. En el mismo sentido la pregunta nueve hace afirmaciones donde el estudiante debe analizar la posibilidad de ocurrencia de una relación. Cuatro niños contestan acertadamente, los otros niños no lo hacen y se concluye que es porque los dos animales no son de la misma especie.

En la décima pregunta el niño debe confirmar la falsedad o veracidad de la afirmación, siete de los diez niños no contesta de manera adecuada, se deduce que los niños presentan confusión en el lenguaje utilizado por el autor del texto. Caso contrario sucede con las preguntas 11 y 12 , a pesar de que es confusa la afirmación de las preguntas, siete niños las contestan acertadamente, esto implica que ellos pueden identificar detalles concretos de la lectura e interpretarlos de manera efectiva, de igual manera reconocen información expresada en el texto de manera clara.

La pregunta trece muestra una afirmación que trae consigo una consecuencia, la mitad de los niños interpretan adecuadamente el propósito de la pregunta, esto implica que los niños realizan inferencias contrarias a lo que se expresa en el texto.

El segundo momento de la prueba diagnóstica consistió en diez preguntas abiertas, el análisis se realizó teniendo en cuenta el desarrollo del cuento. La primera pregunta indaga en los niños acerca de los personajes del cuento; solo la mitad de los que participan de la prueba logra recordar la mayoría de los personajes, los demás nombran solo los personajes principales. La segunda pregunta cuestiona a los niños acerca del porqué él bebe delfín se encontraba triste, a la cual todos los niños emiten una respuesta acertada de forma literal.

De la tercera pregunta que cuestiona a los niños acerca del consejo que recibió el delfincito para encontrar a la madre, ocho de los niños responden de manera adecuada y en forma literal de la lectura; entre tanto la cuarta pregunta que indaga acerca de lo que le paso al delfincito para dejarse ir al fondo del mar, la mayoría de estudiantes narra situaciones adversas, pero no identifican qué realmente ocurrió cuando el pececito cayó al fondo del mar; es decir no hacen uso de argumentos. 
La quinta y sexta pregunta cuestionan acerca de la manera como se sintió el pececito en el fondo del mar y quién acude en su ayuda y por lo menos lo hace reír; la mitad de los niños infieren como se sentía el pececito, pero no logran identificar la respuesta correcta, mientras que la respuesta de la pregunta seis, aunque todos identifican quién lo hizo reír algunos no describen cómo. Esto conduce a pensar que los niños decodifican una lectura, pero no le colocan la suficiente atención.

La pregunta siete es de carácter espacio temporal, cuestiona a los niños acerca de cómo fue la tarde en que apareció el arco iris, en su mayoría, los estudiantes, describen otros sucesos, pero no dan respuesta a lo preguntado; lo que significa que no se evidencia comprensión y apropiación de lo leído. La pregunta ocho hace referencia a cómo era delfincito cuando encuentra a la mamá, la mayoría de los niños contesta acertadamente sobre el estado físico y emocional del pez. Cabe anotar que la pregunta se presta para varias interpretaciones, puede ser que interroguen al niño para que haga una descripción física, o para que cuente el estado emocional del animal, o hay variadas interpretaciones que el niño puede realizar.

Las preguntas nueve y diez cuestionan al estudiante en caso de que en la lectura el personaje central no fuera el delfincito, sino el estudiante; es decir cambiar el roll de lector a actor en la lectura y cuestionarse sobre lo que hubiese hecho en tal caso. En la respuesta a la pregunta nueve se puede ver que identifican la enseñanza de la lectura y en la diez continúan relatando el tema del texto, lo relacionan con la pérdida de la mamá, pero no contestan que harían si fueran ellos.

Para el tercer momento

Primera solicitud: redactar un texto describiendo la situación actual de los delfines en aguas colombianas.

Los diez niños expresan de forma sutil y corta su preocupación por el maltrato del ser humano a los peces del mar y a los animales en general, sin embargo, las ideas son repetidas y desorganizadas. Un niño hace una narración o resumen del cuento no propone ideas nuevas. La falencia más notoria en la producción escrita de los niños se presentó en el uso de conectores, preposiciones, vocabulario, y en el uso adecuado de los signos de puntuación. En el mismo sentido las dificultades presentadas en cohesión entre las palabras para darle sentido a los escritos, y la poca fluidez verbal, no tienen, por así decirlo un gran repertorio de palabras que logren significar lo mismo para evitar la repetición de las mismas.

Segunda solicitud: Ilustrar mediante dibujo y describir lo que más le llamó la atención de la lectura.

La expresión de los dibujos o escenas están llenos de creatividad y colorido, pero la descripción, al igual que el anterior punto, se hacen de forma literal al igual que la explicación. En los textos escritos por los estudiantes se nota gran dificultad en el uso de los signos de puntuación y esto hace 
que sus producciones no establezcan relaciones lógicas entre los enunciados.

Tercera solicitud: Cambiar el final del cuento.

Ningún estudiante cambia el final del cuento, se limitan a escribir uno o dos renglones continuando el final que trae el cuento. Y muchos dan ideas que se prestan para repetir lo ya enunciado. Los estudiantes presentan falencias en la incorporación de nuevas acciones, no presentan fortalezas en aspectos de creatividad e inventiva, quizá por la presión o por evitar equivocaciones $\mathrm{y}$ burlas.

En el análisis de los tres momentos se puede verificar, entre otras, las siguientes situaciones; a pesar de que las respuestas en el primer momento no son del todo equivocadas, se deja ver que los estudiantes no están en condiciones de identificar la pregunta en el texto, no logran recordar acertadamente los detalles de la lectura, no relacionan el final del cuento con la pregunta, presentan cierta confusión de términos, no relacionan y no logran interpretar detalles minuciosos del cuento. En el segundo momento se identifica que los niños no logran recordar todos los personajes del cuento, están en condiciones de analizar las narrativas, pero no identifican lo que sucede, no logran hacer las narraciones que se encuentran en el texto $y$, en el tercer momento que era la narración textual los niños no están en condiciones de escribir párrafos, porque no tienen dominio en conectores, proposiciones, producción escrita, uso de vocabulario y signos de puntuación.

\section{Conclusiones}

Con el análisis de los resultados de la prueba diagnóstica, los docentes manifiestan que la mayoría de sus estudiantes tienen muchas dificultades para leer y comprender textos; así como también quedó demostrado que el proceso de lectura lo hacen los estudiantes por necesidad no por gusto. En la mayoría de sedes, y en los hogares no se crean hábitos de lectura.

De la prueba diagnóstica se pudo concluir que la mayoría de estudiantes hacen lectura superficial, pues al ser interrogados con preguntas sencillas de falso o verdadero, no relacionan el contenido del texto con lo que se pregunta, y hay mayor dificultad cuando la pregunta hace referencia a lectura inferencial. Cuando se hace pregunta abierta el estudiante generalmente responde con monosílabos.

En ese sentido y apuntando a una reflexión docente se hizo necesario retomar la conclusión de la profesora García de Morán donde señala que: "Los alumnos no saben leer ni escribir porque los docentes no saben hacerlo y éstos no lo hacen porque los programas estaban estructurados en términos de conductas y contenidos y no en términos de procesos, por este motivo los dos repiten mecánicamente, no comprenden, no interpretan, no valoran, no crean y no enjuician” (García, 1998).

Los estudiantes disfrutan de las actividades que se programan, se puede ver que a pesar de que se hace la recopilación muchos de los niños lo toman de manera jocosa y al 
representarlo lo hacen de forma literal, se dificulta ir más allá y hacer un análisis crítico de cada situación expuesta.

Se concluyó que el uso de los talleres permitió fortalecer procesos de lectura comprensiva y producción textual; y a través de ellos se incentivó a los estudiantes a que fomenten hábitos de lectura desde la cultura de su contexto. Las actividades fueron bien recibidas por los estudiantes y padres de familia quienes participaron activamente en la recuperación de literatura tradicional como creencias, dichos, refranes y toponimias. Además, se encontró una gran riqueza cultural de tradición popular que era desconocida por los niños objetos de muestra.
El uso de las TIC como herramienta de apoyo posibilitó en los estudiantes mayor motivación, participación y gusto por el desarrollo de cada una de las actividades propuestas, se pudo evidenciar la creatividad y el agrado que causa en los niños poder expresar sus ideas de otra forma.

Las salidas pedagógicas permitieron que los estudiantes interactuaran con los abuelos vecinos, quienes con mucho agrado compartieron sus vivencias, experiencias, historia de vida, anécdotas y a la vez hicieran reflexión sobre la importancia del buen trato el respeto y fortalecimiento de lazos de amistad.

\section{Referencias}

Betancourth, M. \& Madroñero, E. (2004). La enseñanza para la comprensión como didáctica alternativa para mejorar la interpretación y producción oral y escrita en lengua castellana en el grado quinto del centro educativo municipal La Victoria de Pasto (Tesis de Maestría), Universidad de Manizales, Pasto Colombia.

Cassany, D. (1993). Reparar la escritura: Didáctica de la corrección de lo escrito. Editorial Grao

Duarte, R. (2012). La enseña de la lectura como proceso interrelacioado al desarrollo del comportamiento lecto (Tesis Doctoral), Universidad de Alcalá, España.

Elliot, J. (1993). El cambio educativo desde la investigación-acción. Madrid: Morata. García, J. (1998). Hacia un diseño instruccional de lectura. CoroFalcón, Endil-98.

González, R. (2011). Transformaciones en la producción de textos en niños de $2^{\circ}$ de básica primaria através de la integración de las TIC (Tesis de Maestría), Universidad de la Sabana, Bogotá, Colombia.

Latorre, A. (2003). La investigación acción. Conocer y cambiar la practica educativa . España: Graó. 
Legrand, L. (2000). Celetín Freinet (1896-1966). Perspectivas: Revista trimestral de educación comparada, (1-2), 425-441

Madero, I. y. (2013). El proceso de comprensión lectora en alumnos de tercero de secundaria. Revista Mexicana de investigación educativa, 112-139.

Orrego, J., \& Toro, L. (2014). Relaciones vitales: el aula como escenario permanente de investigación. Praxis \& Saber, 5(10), 121 - 139. https://doi. org/10.19053/22160159.302

Vygotsky, L. y Piaget, J. (2012). Teorías del aprendizaje. Madrid: Ariel 